PROCEEDINGS OF THE

AMERICAN MATHEMATICAL SOCIETY

Volume 125, Number 10, October 1997, Pages 3099-3106

S 0002-9939(97)04235-4

\title{
ON THE LENGTHS OF CLOSED GEODESICS ON A TWO-SPHERE
}

\author{
NANCY HINGSTON
}

(Communicated by Christopher Croke)

\begin{abstract}
Let $c$ be an isolated closed geodesic of length $L$ on a compact Riemannian manifold $M$ which is homologically visible in the dimension of its index, and for which the index of the iterates has the maximal possible growth rate. We show that $M$ has a sequence $\left\{c_{n}\right\}, n \in \mathbb{Z}^{+}$, of prime closed geodesics of length $m_{n} L-\varepsilon_{n}$ where $m_{n} \in \mathbb{Z}$ and $\varepsilon_{n} \downarrow 0$. The hypotheses hold in particular when $M$ is a two-sphere and the "shortest" Lusternik-Schnirelmann closed geodesic $c$ is isolated and "nonrotating".
\end{abstract}

Let $M$ be a compact Riemannian manifold of dimension $n$. For $a \in \mathbb{R}$ let $\Lambda^{a}$ be the space of $H^{1}$ closed curves on $M$ of energy $<\frac{a^{2}}{2}$. In this paper we prove the

Theorem. Let $c$ be an isolated closed geodesic of length $L$ on $M$. Let $\lambda \in \mathbb{Z}^{+}$. Assume

(1) c gives local homology in dimension $\lambda$, i.e.,

$$
H_{\lambda}\left(\Lambda^{L} \cup c, \Lambda^{L}\right) \neq 0 \text {. }
$$

(2) $\operatorname{Index}\left(c^{m}\right) \geq m \lambda+(m-1)(n-1)$ for $m \geq 1$.

Then there is an $m_{0} \in \mathbb{Z}^{+}$and a sequence $\sigma_{m} \downarrow 0$ so that if $m \geq m_{0}$, and if $m$ is odd (or if $n$ and $\lambda$ have different parity), $M$ has a closed geodesic $\gamma_{m}$ with length $\ell \in\left(m L-\sigma_{m}, m L\right)$. It follows that $M$ has infinitely many closed geodesics.

Corollary. Suppose $M$ is a two-sphere; suppose the "shortest" Lusternik-Schnirelmann closed geodesic $c$ is isolated and "nonrotating". Then the above conclusion holds (with $\lambda=1$ ).

Background. See $[2,3,9]$ for a more complete discussion. When $M$ is a twosphere, the theorem of Lusternik and Schnirelmann $[6,11]$ gives three simple (i.e. embedded) closed geodesics. If e.g. $M$ has positive curvature, then associated to each simple closed geodesic $c$ is the Birkhoff map $B$. This is an area-preserving self-map of the closed annulus which describes the geodesic flow on $M$ [3]. Interior periodic points of $B$ give closed geodesics on $M$. An elementary and well-known argument, which some say is due to Birkhoff, shows that $B$ has infinitely many periodic orbits unless $c$ is "nonrotating", i.e. unless there is a point on $c$ whose second conjugate point along $c$ occurs after exactly one circuit about $c[3,9,13]$.

For each simple closed geodesic $c$ on a two-sphere $M$ we have three cases:

(i) $B$ is defined, $c$ not nonrotating.

Received by the editors April 2, 1996.

1991 Mathematics Subject Classification. Primary 58E10; Secondary 53C22.

(C)1997 American Mathematical Society 
(ii) $B$ is defined, $c$ nonrotating.

(iii) $B$ is not defined.

As mentioned above, $M$ has infinitely many closed geodesics in case (i). Victor Bangert has proved [2] that there are infinitely many in case (iii) and John Franks [5] that there are infinitely many in case (ii). In [9] we gave an elementary variational proof that $M$ has infinitely many closed geodesics if the longest of the three Lusternik-Schnirelmann geodesics is nonrotating. This followed from the

Complementary Theorem ([9]). Let $c$ be an isolated closed geodesic on a compact Riemannian manifold of dimension n. Let $k \in \mathbb{Z}^{+}$. Assume

1) c gives local homology in dimension $k$.

2) (Index + nullity $)\left(c^{m}\right) \leq m k-(m-1)(n-1)$ for $m \geq 1$.

Then there is an $m_{0} \in \mathbb{Z}^{+}$and a sequence $\sigma_{m} \downarrow 0$ so that if $m \geq m_{0}$, $M$ has a closed geodesic $\gamma_{m}$ with length $\ell \in\left(m L, m L+\sigma_{m}\right)$. It follows that $M$ has infinitely many closed geodesics.

(The above statement is stronger than that in [9]; however, the above can be inferred from the proof.) In [9] we also showed, using the equivariant Morse complex [8], that in all cases the number $N(\ell)$ of closed geodesics of length $\leq \ell$ on a twosphere $M$ satisfies

$$
\liminf N(\ell) \frac{\log \ell}{\ell}>0
$$

The present theorem does nothing to improve this estimate.

One does not expect an analogous theorem about the "middle" LusternikSchnirelmann geodesic; it seems that this one could be nonrotating but nondegenerate and hyperbolic.

Under the hypotheses of the theorem, the index of $c^{m}$ has the fastest possible growth; a geodesic satisfying the hypothesis of the complement has the slowest possible growth. The idea of the theorem is the same as that of the complement but the Morse theory is "upside down". (The proof, however, is quite different.) In the complement the local negative cycle coming from $c^{m}$ is "locally" homologous to a shorter cycle, while in the theorem the local homology class of the dual positive cycle is "locally" homologous to a longer cycle. The $\gamma_{m}$ are "near" $c c^{m}$ in the sense that $\gamma_{m}$ lies in the closure of the stable (resp. unstable) manifold of $c^{m}$. We think it is remarkable that in both cases we do not seem quite to "catch" the geodesics $\gamma_{m}$, but only see their shadows. (In the theorem this shadow is a point in a tubular neighborhood of $c^{m}$ in the unstable manifold of $\gamma_{m}$.) In particular: The estimate for $N(\ell)$ mentioned above uses a "divisibility" result for surfaces [8]. In both the theorem and the complement it seems obvious, but does not quite follow from the proof, that the geodesic $\gamma_{m}$ lies in a tubular neighborhood of $c_{m}$. If we could prove that this were the case, the multiplicity of $\gamma_{m}$ would be a divisor of $m$ (a stronger version of divisibility) and the growth estimate would follow immediately. That $\gamma_{m}$ could move far enough away from $c^{m}$ to develop a stability group which is not a subgroup of $\mathbb{Z}_{m}$ we find almost unbelievable. We invite the reader to try to do a better job of nabbing the $\gamma_{m}$.

In both the theorem and the complement the local homology of $c^{m}$ becomes "unstable" as $m \rightarrow \infty$. It appears that if one were to stand back a little from $M$ and squint one would "see" a closed geodesic with nondegenerate-type index growth instead of $c$. 
Note that, given condition (2), (1) is equivalent to the weaker hypothesis that $H_{\lambda}\left(\Lambda^{L} \cup S^{1} \cdot c, \Lambda^{L}\right) \neq 0$. Using the theorem, Birkhoff's result [3] and the elementary argument found in $[13,9]$ (which may or may not have been known to Birkhoff), one can prove that a metric of positive curvature on $S^{2}$ has infinitely many closed geodesics provided that Birkhoff's "minimax" geodesic has no self-intersections.

We first prove that the corollary follows from the theorem. Next we give a rough sketch of the proof of the theorem, and finally we prove the theorem.

Proof of Corollary (from the Theorem). The "shortest" Lusternik-Schnirelmann geodesic can be found by taking the minimax, using an appropriate curve shortening process [6], of the homology class in the space of unparameterized embedded curves on $M$ represented by the image of the one-parameter family of circles on the standard sphere $S^{2}$ parallel to a fixed great circle under a diffeomorphism $S^{2} \rightarrow M$. This produces a simple closed geodesic $c$ with local homology in dimension 1 [1]. If $c$ is nonrotating, then index $\left(c^{m}\right) \geq 2 m-1$ (counting conjugate points) and thus (1) and (2) hold with $\lambda=1$.

Sketch of proof of the theorem. We could not find a proof of the theorem as simple as that of the complement. To give the idea of the proof we first give a rough sketch in a (not so) special case. Condition (2) implies that the Poincaré map $P$ of $c$ satisfies $(P-1)^{2}=0$. In the case when the kernel of $(P-1)$ has dimension $n-1$ (the least degenerate case), we can choose a point $Q=c(0)$ which is not conjugate to $c(1)$ along $c$. In this case the proof is much simpler. Let $W$ be a local transverse hypersurface to $c$ at $Q$. If $\Omega_{W}$ is a neighborhood of $c$ in the set of curves starting and ending on $W$, we have the fibration

$$
f: \Omega_{W} \rightarrow W \times W .
$$

The inverse image $\Lambda_{W}$ of the diagonal $\Delta$ gives a neighborhood of $c$ in $\Lambda$ and the inverse image of $o$ gives a neighborhood of $c$ in the based loop space $\Omega_{Q}$. Now $c$ has index $\lambda$ in $\Omega_{Q}$ and in $\Lambda$. In the "special case" each fiber of $f$ contains a unique geodesic, also of index $\lambda$; thus "Morse theoretically"

$$
\Omega_{W} \stackrel{\sim}{\rightarrow} W \times W \times V
$$

where $V$ is a disk of dimension $\lambda$ and where on each fiber $w_{0} \times w_{1} \times V$, the length $\ell$ has a nondegenerate local maximum at the origin $x=0$ in $V$. Moreover $\ell$ has a degenerate strict local minimum at the origin in $\Delta \times o$. Similarly, for a neighborhood $\Omega_{m}$ of $c^{m}$ in $\Lambda$, we have

$$
\Omega_{m} \stackrel{\sim}{\rightarrow} W^{m} \times V^{m}
$$

since a curve in $\Omega_{m}$ has $m$ "pieces", each of which lies in $\Omega_{W}$. Now $W^{m} \times V^{m}$ has dimension $m(n-1)+m \lambda$, and length increases in the direction of $\Delta \times o \subseteq W^{m} \times V^{m}$. Since $\Delta \times o$ has dimension $n-1$, and index $\left(c^{m}\right)=(m-1)(n-1)+m \lambda$, a maximal negative disk $N$ for $o$ in $W^{m} \times V^{m}$ must be transverse to $\Delta \times o$ at 0 . However, $N$ cannot avoid the higher dimensional set $\Delta^{\prime} \times o$ where

$$
\Delta^{\prime}=\left\{w_{0}=\cdots=w_{j} ; w_{j+1}=\cdots=w_{m}\right\}, \quad j \approx m / 2 .
$$

Curves in $\Delta^{\prime} \times o$ have $m-2$ "long" pieces and only 2 "short" pieces. For large $m$, $(m-2)$ times a degenerate minimum will win out quickly over twice a nondegenerate maximum, and the boundary sphere $S$ of $N$ cannot be pushed much below the level $m L$. 
Proof of the theorem. Conditions (1) and (2) imply that $c$ has index $\lambda$. Let $r$ be the injectivity radius of $M$. Let $k \in \mathbb{Z}^{+}$with $L / k<r / 8$. Let $W=W_{0}, W_{1}, \ldots, W_{k}$, $W_{k+1}=W$ be closed $(n-1)$ disks transverse to $c$ at the $c(i / k+1)$. Let

$$
\begin{aligned}
& \Omega_{W}=W \times W_{1} \times \cdots \times W_{k} \times W, \\
& \quad f: \Omega_{W} \rightarrow W \times W, \\
& \Lambda_{W}=f^{-1}(\Delta), \quad \Lambda_{W} \approx W \times W_{1} \times \cdot \times W_{k},
\end{aligned}
$$

where $\Delta \subseteq W \times W$ is the diagonal, and

$$
\begin{aligned}
& \Omega_{Q}=f^{-1}(0), \quad \Omega_{Q} \approx W_{1} \times \cdots \times W_{k}, \\
& \Omega_{m}=\left(W \times W_{1} \times \cdots \times W_{k}\right)^{m} .
\end{aligned}
$$

Each of the above is identified with a set of curves: $\Omega_{W}$ is identified with the set of piecewise geodesics starting and ending on $W$ in the obvious way. $\Lambda_{W} \subset \Omega_{W}$ is the subset of closed curves, and $\Omega_{Q} \subset \Lambda_{W}$ the closed curves at $Q=c(0)$. $\Omega_{m}$ is in the obvious way identified with a set of closed curves near $c^{m} ; \Omega_{m}$ is the subset of $\Omega_{W}^{m}$ given by incidence relations on the ends. The natural function on these spaces is length. Thus, e.g. $\Omega_{m}^{a} \subseteq \Omega_{m}$ is the subset of curves of length $<a$.

If $\eta$ is the nullity of $c$, the length function has a critical point at the origin in $\Lambda_{W}$ of index $\lambda$ and nullity $\eta$. (This follows from arguments in [12] although our finite dimensional approximation is somewhat different, corresponding to a neighborhood of $c$ in the space of unparameterized curves.) The space $\Lambda_{W}$ carries the local topology of $\Lambda$ : If $U$ is a sufficiently small $H^{1}$ neighborhood of $c$ in $\Lambda$, we get a continuous length decreasing map $U \rightarrow \Lambda_{W}$ as follows. Let $N$ be such that $L / N<r / 2 \leq L / N+1$. If $\gamma \in U$ has length $\ell$, first divide $\gamma$ into pieces of length $\ell / N$. Next replace each such piece by a geodesic segment. If $U$ is sufficiently small, the endpoints of these pieces will lie close to the points $c(i / N)$ and the curve will now intersect the $W_{i}$ exactly once transversally; thus we have a retraction onto $\Lambda_{W}$. Thus in particular $\Lambda_{W}$ contains a representative of the local homology class of $c$. After shrinking the disks $W_{i}$ we can find coordinates $(x, y, z) \in D^{\lambda} \times$ $D^{(k+1)(n-1)-\lambda-\eta} \times D^{\eta}$ on $\Lambda_{W}$ so that $O$ is the only critical point of $\ell$ in $\Lambda_{W}$ and so that [7]

$$
\ell=L-x^{2}+y^{2}+g(z) .
$$

Since the critical point has local homology in dimension $\lambda, g$ has a strict local minimum at $O$. (The complement proof in [9] contains the proof of a similar fact.)

Let $w$ be a coordinate on $W$ and thus on $\Lambda_{W}$. Then

A) Given $u>0 \exists \rho_{u}>0$ so that

$$
\ell>L+\rho_{u} \text { on }\{|w| \geq u ; x=0\} \subseteq \Lambda_{W} .
$$

B) $\exists \mu>0$ so that $\{\ell \leq L+\mu ; x=0\}$ lies in the interior of $\Lambda_{W}$.

We can assume $\rho_{u}$ increases with $u$.

We now move from $c$ to $c^{m}$. Let $\Omega_{m}$ lie in the interior of a slightly larger version $\widetilde{\Omega}_{m}$ (extending the $W_{i}$ ). We define a map $\psi_{0}$ from an $H^{1}$ neighborhood $U$ of $c^{m}$ into $\widetilde{\Omega}_{m}$ as follows: Let $N$ be such that $m L / N<r / 2 \leq m L / N+1$. Given $\gamma \in U$, as before replace $\gamma$ by $N$ geodesic segments and then retract to $\widetilde{\Omega}_{m}$. We claim that, if the $W_{i}$ and $\varepsilon>0$ are sufficiently small (independent of $m$ ), $\psi_{0}$ extends to a continuous map

$$
\psi: \Lambda^{m L+\varepsilon} / \Lambda^{m L-\varepsilon} \rightarrow \Omega_{m} / \partial \Omega_{m} \cup \Omega_{m}^{m L-\varepsilon} ; \mathbb{Z}_{m} .
$$


Here $\mathbb{Z}_{m}$ acts by permutations; we need to divide by the $\mathbb{Z}_{m}$-action since we allow all possible parameterizations in $\Lambda^{m L+\varepsilon}$. The map $\psi$ does not increase length on points not mapped to the basepoint.

Proof. We expect $\varepsilon \ll r$. Assume $U$ is a maximal open subset of $\Lambda$ on which $\psi_{0} / \mathbb{Z}_{m}$ is defined. The map $\psi_{0}$ consists of two deformations. The first is always defined on $\Lambda^{m L+\varepsilon}$, and results in an intermediate curve which is short or which consists of $N$ geodesic segments of length $\geq r / 4$. If the $W_{i}$ are sufficiently small, and if the intermediate curve meets each $W_{i}$ transversally (in cyclic order) exactly $m$ times, and is not significantly shortened after the second deformation, its pieces lie $\mathcal{C}^{1}$ close to $c^{m}$ and thus the intermediate curve lies in the interior of the set where the second deformation is defined $\bmod \mathbb{Z}_{m}$. Thus, if $\varepsilon$ and the $W_{i}$ are small,

$$
\left\{\gamma \mid \gamma \in U \cap \Lambda^{m L+\varepsilon} ; \psi_{0} / \mathbb{Z}_{m}(\gamma) \in \Omega_{m} / \mathbb{Z}_{m} ; \ell \psi_{0}(\gamma) \geq m L-\varepsilon\right\}
$$

is a closed set in the interior of $U$. Any point for which the second deformation is not defined maps to the basepoint. Since $\psi_{0}$ is continuous on $U, \psi$ is continuous. From now on the $W_{i}$ and $\rho_{u}, \mu, \varepsilon$ are fixed; we assume that the coordinates $(x, y, z)$ are defined on $\Lambda_{W}$ and that $\psi$ is defined.

Next we construct a map

$$
\begin{aligned}
\varphi: \Omega_{m} & \rightarrow W^{m} \times V^{m} \\
\gamma & \mapsto\left(w_{1}, \ldots, w_{m}, x_{1}, \ldots, x_{m}\right)
\end{aligned}
$$

where $V \approx D^{\lambda}$ is the range of the coordinate $x$ on $\Lambda_{W}$. The $w_{i}$ are the $m$ intersections of $\gamma$ with $W$. The curve $\gamma$ has $m$ "pieces", each in $\Omega_{W}$. Let

$$
p: \Omega_{W} \rightarrow \Lambda_{W} \approx W \times W_{1} \times \cdots \times W_{k}
$$

by averaging the two coordinates on $W$; then $x \circ p$ on each piece of $\gamma$ gives $x_{1}, \ldots, x_{m}$. This step identifies the fiber of $f$. Suppose $\tau \in \Omega_{W}$ has coordinates $\left(w_{0}, w_{1}, x\right) \in$ $W \times W \times V($ by $x$ we mean $x \circ p)$. Then

$$
\begin{aligned}
& \ell(\tau)+\left|w_{1}-w_{0}\right| \geq \ell \circ p(\tau) . \\
& \ell \circ p(\tau) \geq L \quad \text { if } x(\tau)=0 .
\end{aligned}
$$

C) follows from the triangle inequality if we choose a norm on $W$ that exceeds the arclength on $W$.

Finally project $W^{m} \rightarrow \overline{W^{m}}$ where $\overline{W^{m}} \approx D^{(n-1)(m-1)}$ is the orthogonal complement $\sum w_{i}=0$ of the diagonal $\Delta$ in $W^{m}$. This gives

$$
\bar{\varphi}: \Omega_{m} \rightarrow \overline{W^{m}} \times V^{m}
$$

Let $j \leq m / 2$ but $j \approx m / 2$ and let

$$
\Delta^{\prime}=\left\{w_{i}=\cdots=w_{j} ; w_{j+1}=\cdots=w_{m}\right\} \subseteq \overline{W^{m}}
$$

For simplicity assume $m=2 j$. (This is impossible if $m$ is odd, but close to the truth if $m$ is large.) The inverse image of a point $(w, \ldots, w,-w, \ldots,-w) \in \Delta^{\prime}$ consists of points $(z+w, \ldots, z+w, z-w, \ldots, z-w) \in W^{m}$ for $z \in W$. Putting together A), C), and D), we have

$$
\ell \geq m L+(j-1) \rho_{|w|}-4|w| \text { on } \bar{\varphi}^{-1}\left(\Delta^{\prime} \times 0\right),
$$

where $w$ is the coordinate on $\Delta^{\prime}$. (Of the $m$ pieces of a curve in $\bar{\varphi}^{-1}\left(\Delta^{\prime} \times 0\right)$, two have length $\geq L-2|w|$ by C), D); $j-1$ have length $\geq L+\rho_{|z+w|}$ and $m-j-1$ have length $\geq L+\rho_{|z-w|}$ by A). Either $\rho_{|z+w|}$ or $\rho_{|z-w|}$ is $\geq \rho_{|w|}$.) 
Similarly, combining A), B), C), and D), if $W$ contains a disk of radius $\eta$, we have

$\mathrm{B})^{*} \quad \ell \geq \min \left\{\begin{array}{l}m L+\mu-4|w| \\ m L+(j-1) \rho_{\eta}-4|w|\end{array} \quad\right.$ on $\bar{\varphi}^{-1}\left(\Delta^{\prime} \times 0\right) \cap \partial \Omega_{m}$.

(The second estimate holds if $z+w$ or $z-w \in \partial W$.)

Pick $\delta>0$ but with $\delta<\min \left(\eta, \frac{\mu}{4}, \frac{\varepsilon}{4}\right)$. Next find $m_{0}$ so that for $m \geq m_{0}$ we have

$$
(j-1) \rho_{\delta}>4 \delta \quad\left(j=\frac{m}{2} \text { or } \frac{m-1}{2}\right) .
$$

Then also $(j-1) \rho_{\eta}>4 \delta$. Let $\Delta_{\delta}$ be the subset of $\Delta^{\prime} \times 0$ where $|w| \leq \delta$. By A $)^{*}$

$$
\ell>m L-\varepsilon \text { on } \bar{\varphi}^{-1}\left(\Delta_{\delta}\right)
$$

By B)*

$$
\ell>m L \quad \text { on } \partial \Omega_{m} \cap \bar{\varphi}^{-1}\left(\Delta_{\delta}\right)
$$

Thus, if

$$
B=\operatorname{closure} \bar{\varphi}\left(\Omega_{m}^{m L} \cap\left(\partial \Omega_{m} \cup \Omega_{m}^{m L-\varepsilon}\right)\right),
$$

$B$ does not meet $\Delta_{\delta}$. Combining $\psi$ and $\bar{\varphi}$, we get a continuous map for $m \geq m_{0}$

$$
\Psi: \Lambda^{m L} / \Lambda^{m L-\varepsilon} \rightarrow \overline{W^{m}} \times V^{m} / B ; \mathbb{Z}_{m}
$$

Now let $N_{m}$ be a maximal negative definite subspace of the Hessian form on $T_{c^{m}} \Omega_{m}$. Consider

$$
d \varphi: T_{c^{m}} \Omega_{m} \rightarrow T_{0}\left(W^{m} \times V^{m}\right) .
$$

The kernel of the map $T_{c^{m}} \Omega_{m} \rightarrow T_{0} W^{m}$ is naturally $\left(T_{c} \Omega_{Q}\right)^{m}$. Since a negative definite vector in $T_{c} \Omega_{Q}$ cannot map to 0 in $T_{0} V, N_{m}$ injects into $T_{0}\left(W^{m} \times V^{m}\right)$. Now $\operatorname{dim}\left(W^{m} \times V^{m}\right)=\operatorname{dim} N_{m}+\operatorname{dim}(\Delta \times 0)$. By A $)^{*}, \ell \geq m L$ on $\varphi^{-1}(\Delta \times 0)$ and thus

$$
d \bar{\varphi}: N_{m} \stackrel{\sim}{\rightarrow} T_{0}\left(\overline{W^{m}} \times V^{m}\right)
$$

is an isomorphism. Applying the exponential map to a sufficiently small sphere of codimension 1 in $N_{m}$ gives a sphere $S$ in $\Omega_{m}^{m L-\kappa}$ for some $\kappa>0$ whose image in $\overline{W^{m}} \times V^{m}$ is a sphere encircling 0 . We can assume that the image of $S$ in $\overline{W^{m}}$ lies at a distance $<\delta$ from the origin, and that $\kappa<\varepsilon$. (In fact, the latter follows from the former.)

Now let $m \geq m_{0}$ and $R \in \partial \Delta_{\delta}$. By C), D) we can find a disk $D$ about the origin in $\overline{W^{m}} \times V^{m}$ with $\ell>m L-\kappa$ in $\bar{\varphi}^{-1}(D)$. (Near the origin in $\overline{W^{m}}$ the term $\left|w_{1}-w_{0}\right|$ in C) is small.) By shrinking $D$, we can assume $R \notin D$ and $D \cap B=\varnothing$, since $B$ does not meet $\Delta_{\delta}$. Let $\overline{O R}$ denote the segment in $\Delta_{\delta}$ from the origin to $R$. Let $D^{\prime}$ be a disk in $\overline{W^{m}} \times V^{m}$ with

$$
\overline{O R} \subset \operatorname{Interior}\left(D \cup D^{\prime}\right) .
$$

We can assume $D^{\prime} \cap B=\varnothing$ and that $\mathbb{Z}_{m} \times D^{\prime}$ consists of $m$ disjoint sets. (The latter is possible since $\mathbb{Z}_{m}$ acts freely on $\Delta^{\prime}$ except at 0 .) Then $D \cup D^{\prime} \rightarrow \overline{W^{m}} \times V^{m}$ induces a continuous map

$$
\overline{W^{m}} \times V^{m} / B \rightarrow D \cup D^{\prime} / \partial\left(D \cup D^{\prime}\right) .
$$




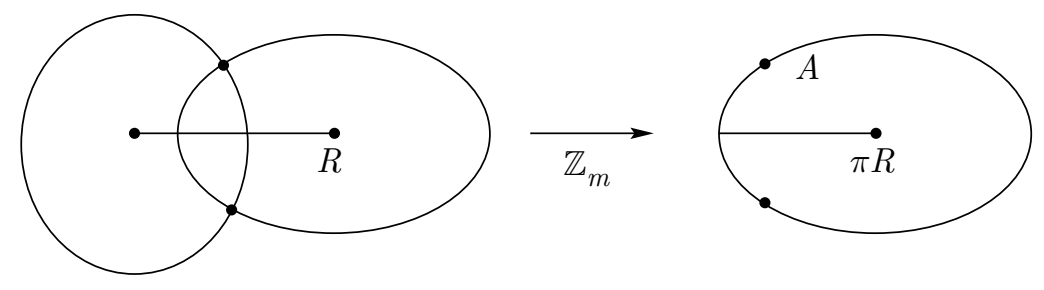

FiguRE 1

By A)* and E), $\ell>m L$ on $\bar{\varphi}^{-1}(R)$. Combining the above map with $\Phi$ gives a continuous map

$$
\Psi: \Lambda^{m L-\kappa} / \Lambda^{m L-\varepsilon} \rightarrow A / B^{\prime}
$$

where

$$
\begin{aligned}
A & =\operatorname{Image}\left(D^{\prime} \backslash\{R\}\right) \subset \overline{W^{m}} \times V^{m} / \mathbb{Z}_{m} ; \\
B^{\prime} & =\operatorname{Image}\left(\partial D^{\prime} \cap \partial\left(D^{\prime} \cap D\right)\right) \subset A .
\end{aligned}
$$

Topologically $A / B^{\prime}$ is a sphere with two holes which are joined by the image of the segment $\overline{O R}$. (See Figure 1.)

Now $\mathbb{Z}_{m}$ will preserve orientation on $\overline{W^{m}} \times V^{m}$ if $m$ is odd or if $n$ and $\lambda$ have different parity. Assume this is the case. The image of $S$ in $\overline{W^{m}} \times V^{m}$ has intersection number $m$ with $\mathbb{Z}_{m} \times \overline{O R}$, and thus the image of $S$ in $A / B^{\prime}$ has intersection number $m$ with the image $\pi \overline{O R}$ of $\overline{O R}$. If $S$ and $S^{\prime}$ are homotopic in $\Lambda^{m L-\kappa}$, then $\Psi\left(S^{\prime}\right)$ must meet $\pi \overline{O R}$. Thus, by $\left.\mathrm{A}\right)^{*}$, the minimax value for the homotopy class of $S$ in $\Lambda^{m L-\kappa}$ is at least $m L-\sigma_{m}$, where $\sigma_{m}$ is the maximum value of

$$
4 u-(j-1) \rho_{u} \text { for } u \in[0, \delta] .
$$

Clearly $\sigma_{m} \rightarrow 0$ as $m \rightarrow \infty$. This minimax value will be achieved by a closed geodesic $\gamma_{m}$. Note that $\gamma_{m}$ lies in the unstable manifold of $c^{m}$, and that some point in $\Psi^{-1}(\pi \overline{O R})$ lies in the unstable manifold of $\gamma_{m}$.

Finally, we show that the sequence $\gamma_{m}$ must contain an infinite number of geometrically distinct closed geodesics. Let $\tau$ be a prime closed geodesic of length $\ell$. If $L / \ell \in \mathbb{Q}$, then there is a constant $\varepsilon>0$ so that

$$
|m L-k \ell|>\varepsilon \quad \text { if } m L \neq k \ell .
$$

Thus, only a finite number of the iterates $\tau^{k}$ could appear among the $\gamma_{m}$. Now suppose $L / \ell \notin \mathbb{Q}$. Given $N \in \mathbb{Z}$, let

$$
\varepsilon_{N}=\min _{\substack{1 \leq m \leq N \\ k \in \mathbb{Z}}}|m L-k \ell|>0 .
$$

Assume $p$ is large enough so that $\sigma_{m}<\varepsilon_{N}$ for $m \geq p$; assume $q>p$ and that $\gamma_{p}$ and $\gamma_{q}$ are both iterates of $\tau$. Then for some $k, j \in \mathbb{Z}$,

$$
0<p L-k \ell<\sigma_{p} \quad \text { and } \quad 0<q L-j \ell<\sigma_{q}
$$

so that

$$
|(q-p) L-(j-k) \ell|<\varepsilon_{N},
$$

which implies $q-p>N$. Thus, the frequency with which the iterates of $\tau$ appear among the $\gamma_{m}$ goes to 0 . 


\section{REFERENCES}

1. W. Ballmann, G. Thorbergsson, and W. Ziller, Existence of closed geodesics on possibly curved manifolds, J. Differential Geom. 18 (1983), 221-252. MR 84i:58032

2. V. Bangert, On the existence of closed geodesics on two-spheres, International J. of Math. Vol. 4, No. 1 (1993), 1-10. MR 94d:58036

3. G. D. Birkhoff, Dynamical Systems, Amer. Math. Soc. Colloq. Publ. 9, Amer. Math. Soc., Providence, 1927.

4. R. Bott, On the iteration of closed geodesics and the Stern intersection theory, Comm. Pure Appl. Math. 9 (1956), 171-206. MR 19:859f

5. J. Franks, Geodesics on $S^{2}$ and periodic points of annulus diffeomorphisms, Invent. Math. 108 (1992), 403-418. MR 93f:58192

6. M. Grayson, Shortening embedded curves, Ann. of Math. (2) 129 (1989), 71-111. MR 90a:53050

7. D. Gromoll and W. Meyer, On differentiable functions with isolated critical points, Topology 8 (1969), 361-369. MR 39:7633

8. N. Hingston, On the equivariant Morse complex of the free loop space of a surface, preprint, 1991.

9. $\quad$ On the growth of the number of closed geodesics on the two-sphere, Internat. Math. Res. Notices 1993, no. 9, 253-262. MR 94m:58044

10. W. Klingenberg, Riemannian Geometry, de Gruyter Stud. Math. 1, de Gruyter, Berlin, 1982. MR 84j:53001

11. L. Lusternik and L. Schnirelmann, Sur le problème de trois géodésiques fermées sur les surfaces de Genre O, C. R. Acad. Sci. Sér. I Math 189 (1929), 269-271.

12. J. Milnor, Morse Theory, Annals of Math. Studies, Princeton Univ. Press, Princeton, 1969. MR 29:634

13. W. D. Neumann, Generalizations of the Poincaré Birkhoff fixed point theorem, Bull. Austral. Math. Soc. 17 (1977), 375-389. MR 58:28435

Department of Mathematics, The College of New Jersey, Trenton, New Jersey 08650

E-mail address: hingston@tcnj.edu 